PROCEEDINGS OF THE

AMERICAN MATHEMATICAL SOCIETY

Volume 138, Number 10, October 2010, Pages 3689-3701

S 0002-9939(10)10377-3

Article electronically published on May 6, 2010

\title{
SQUARE-MEAN ALMOST AUTOMORPHIC SOLUTIONS FOR SOME STOCHASTIC DIFFERENTIAL EQUATIONS
}

\author{
MIAOMIAO FU AND ZHENXIN LIU \\ (Communicated by Yingfei Yi)
}

\begin{abstract}
The concept of square-mean almost automorphy for stochastic processes is introduced. The existence and uniqueness of square-mean almost automorphic solutions to some linear and non-linear stochastic differential equations are established provided the coefficients satisfy some conditions. The asymptotic stability of the unique square-mean almost automorphic solution in the square-mean sense is discussed.
\end{abstract}

\section{INTRODUCTION}

The concept of almost automorphy is a generalization of almost periodicity. It is introduced by Bochner [5] in relation to some aspects of differential geometry. Almost automorphic functions are characterized by the following property. Let $f$ be a continuous function; given any sequence of real numbers $\left\{s_{n}^{\prime}\right\}$, we can extract a subsequence $\left\{s_{n}\right\}$ such that for some function $g$

$$
g(t)=\lim _{n \rightarrow \infty} f\left(t+s_{n}\right) \quad \text { and } \quad \lim _{n \rightarrow \infty} g\left(t-s_{n}\right)=f(t)
$$

for each $t \in \mathbb{R}$. Almost automorphy has been studied by many authors; see Veech [15, 16, for classical exposition; see Johnson [10], Shen and Yi [12], N'Guérékata [7] for recent developments, among others.

Recently, some authors have studied the almost periodic or pseudo almost periodic solutions to stochastic differential equations; see [2, 3, 4, 6, 9, 13, 14, among others. In this paper, we go one step further by introducing the concept of squaremean almost automorphic stochastic processes. Under some conditions of coefficients, we establish the existence and uniqueness of square-mean almost automorphic solutions for some stochastic differential equations.

The paper is organized as follows. In section 2, we introduce the notion of square-mean almost automorphic processes and study some of their basic properties. In sections 3 and 4 , given some suitable conditions, we prove the existence and uniqueness of square-mean almost automorphic mild solutions to some linear and non-linear stochastic differential equations, respectively. In section 5, we discuss

Received by the editors October 27, 2009 and, in revised form, January 11, 2010.

2010 Mathematics Subject Classification. Primary 60H25, 34C27, 34F05, 34G20.

Key words and phrases. Almost automorphy, stochastic differential equations.

The second author is partially supported by NSFC Grant 10801059, SRFDP Grant 20070183053, the 985 Program of Jilin University, and the science research fund at Jilin University. 
the asymptotic stability property of the unique square-mean almost automorphic solution in the square-mean sense.

\section{Square-mean almost automorphic processes}

Throughout this paper, we assume that $(\mathbb{H},\|\cdot\|)$ is a real separable Hilbert space, $(\Omega, \mathcal{F}, \mathbf{P})$ is a probability space, and $\mathcal{L}^{2}(\mathbf{P}, \mathbb{H})$ stands for the space of all $\mathbb{H}$-valued random variables $x$ such that

$$
\mathbf{E}\|x\|^{2}=\int_{\Omega}\|x\|^{2} d \mathbf{P}<\infty .
$$

For $x \in \mathcal{L}^{2}(\mathbf{P}, \mathbb{H})$, let

$$
\|x\|_{2}:=\left(\int_{\Omega}\|x\|^{2} d \mathbf{P}\right)^{1 / 2} .
$$

Then it is routine to check that $\mathcal{L}^{2}(\mathbf{P}, \mathbb{H})$ is a Hilbert space equipped with the norm $\|\cdot\|_{2}$.

Definition 2.1. A stochastic process $X: \mathbb{R} \rightarrow \mathcal{L}^{2}(\mathbf{P}, \mathbb{H})$ is said to be stochastically continuous if

$$
\lim _{t \rightarrow s} \mathbf{E}\|X(t)-X(s)\|^{2}=0 .
$$

Definition 2.2. A stochastically continuous stochastic process $x: \mathbb{R} \rightarrow \mathcal{L}^{2}(\mathbf{P}, \mathbb{H})$ is said to be square-mean almost automorphic if every sequence of real numbers $\left\{s_{n}^{\prime}\right\}$ has a subsequence $\left\{s_{n}\right\}$ such that for some stochastic process $y: \mathbb{R} \rightarrow \mathcal{L}^{2}(\mathbf{P}, \mathbb{H})$

$$
\lim _{n \rightarrow \infty} \mathbf{E}\left\|x\left(t+s_{n}\right)-y(t)\right\|^{2}=0 \text { and } \lim _{n \rightarrow \infty} \mathbf{E}\left\|y\left(t-s_{n}\right)-x(t)\right\|^{2}=0
$$

hold for each $t \in \mathbb{R}$. The collection of all square-mean almost automorphic stochastic processes $x: \mathbb{R} \rightarrow \mathcal{L}^{2}(\mathbf{P}, \mathbb{H})$ is denoted by $A A\left(\mathbb{R} ; \mathcal{L}^{2}(\mathbf{P}, \mathbb{H})\right)$.

In the following lemma, we list some basic properties of square-mean almost automorphic stochastic processes.

Lemma 2.3. If $x, x_{1}$ and $x_{2}$ are all square-mean almost automorphic stochastic processes, then

(1) $x_{1}+x_{2}$ is square-mean almost automorphic.

(2) $\lambda x$ is square-mean almost automorphic for every scalar $\lambda$.

(3) There exists a constant $M>0$ such that $\sup _{t \in \mathbb{R}}\|x(t)\|_{2} \leq M$. That is, $x$ is bounded in $\mathcal{L}^{2}(\mathbf{P}, \mathbb{H})$.

Proof. Since statements (1) and (2) are obvious, we only prove (3). If $\sup _{t \in \mathbb{R}}\|x(t)\|_{2}$ $=\infty$, then there exists a sequence of real numbers $\left\{s_{n}^{\prime}\right\}$ such that

$$
\lim _{n \rightarrow \infty}\left\|x\left(s_{n}^{\prime}\right)\right\|_{2}^{2}=\lim _{n \rightarrow \infty} \mathbf{E}\left\|x\left(s_{n}^{\prime}\right)\right\|^{2}=\infty .
$$

Since $x \in A A\left(\mathbb{R} ; \mathcal{L}^{2}(\mathbf{P}, \mathbb{H})\right)$, there exists a subsequence $\left\{s_{n}\right\} \subset\left\{s_{n}^{\prime}\right\}$ and a stochastic process $y: \mathbb{R} \rightarrow \mathcal{L}^{2}(\mathbf{P}, \mathbb{H})$ such that

$$
\lim _{n \rightarrow \infty} \mathbf{E}\left\|x\left(t+s_{n}\right)-y(t)\right\|^{2}=0 \quad \text { for all } t \in \mathbb{R} .
$$

In particular, when $t=0$ in (2.1), it follows that

$$
\lim _{n \rightarrow \infty} \mathbf{E}\left\|x\left(s_{n}\right)\right\|^{2}<\infty,
$$

a contradiction. 
Theorem 2.4. $A A\left(\mathbb{R} ; \mathcal{L}^{2}(\mathbf{P}, \mathbb{H})\right)$ is a Banach space when it is equipped with the norm

$$
\|x\|_{\infty}:=\sup _{t \in \mathbb{R}}\|x(t)\|_{2}=\sup _{t \in \mathbb{R}}\left(E\|x(t)\|^{2}\right)^{\frac{1}{2}},
$$

for $x \in A A\left(\mathbb{R} ; \mathcal{L}^{2}(\mathbf{P}, \mathbb{H})\right)$.

Proof. By Lemma 2.3 $A A\left(\mathbb{R} ; \mathcal{L}^{2}(\mathbf{P}, \mathbb{H})\right)$ is a vector space, so it is easy to verify that $\|\cdot\|_{\infty}$ is a norm on $A A\left(\mathbb{R} ; \mathcal{L}^{2}(\mathbf{P}, \mathbb{H})\right)$. We only need to show that $A A\left(\mathbb{R} ; \mathcal{L}^{2}(\mathbf{P}, \mathbb{H})\right)$ is complete with respect to the norm $\|\cdot\|_{\infty}$. To this end, assume that $\left\{x_{n}\right\} \subset$ $A A\left(\mathbb{R} ; \mathcal{L}^{2}(\mathbf{P}, \mathbb{H})\right)$ is a Cauchy sequence with respect to $\|\cdot\|_{\infty}$ and that $x$ is the pointwise limit of $x_{n}$ with respect to $\|\cdot\|_{2}$; i.e.

$$
\lim _{n \rightarrow \infty}\left\|x_{n}(t)-x(t)\right\|_{2}=0 \quad \text { for each } t \in \mathbb{R} .
$$

Note that this limit $x$ always exists by the completeness of $\mathcal{L}^{2}(\mathbf{P}, \mathbb{H})$ with respect to $\|\cdot\|_{2}$. Since $\left\{x_{n}\right\}$ is Cauchy with respect to $\|\cdot\|_{\infty}$, the convergence in (2.2) is actually uniform for $t \in \mathbb{R}$. We need to show that $x \in A A\left(\mathbb{R} ; \mathcal{L}^{2}(\mathbf{P}, \mathbb{H})\right)$.

First, we verify that $x$ is stochastically continuous. In fact, by

$x(t+\triangle t)-x(t)=x(t+\triangle t)-x_{n}(t+\triangle t)+x_{n}(t+\triangle t)-x_{n}(t)+x_{n}(t)-x(t)$,

it follows that

$$
\begin{gathered}
\mathbf{E}\|x(t+\triangle t)-x(t)\|^{2} \\
\leq 3 \mathbf{E}\left\|x(t+\triangle t)-x_{n}(t+\triangle t)\right\|^{2}+3 \mathbf{E}\left\|x_{n}(t+\triangle t)-x_{n}(t)\right\|^{2}+3 \mathbf{E}\left\|x(t)-x_{n}(t)\right\|^{2} .
\end{gathered}
$$

By the uniform convergence of $x_{n}$ to $x$ with respect to $\|\cdot\|_{2}$ and the stochastic continuity of $x_{n}$, the stochastic continuity of $x$ follows.

Next, we prove that $x$ is square-mean almost automorphic. Let $\left\{s_{n}^{\prime}\right\}$ be an arbitrary sequence of real numbers; then by standard diagonal progress, we can extract a subsequence $\left\{s_{n}\right\} \subset\left\{s_{n}^{\prime}\right\}$ such that for stochastic processes $y_{i}: \mathbb{R} \rightarrow$ $\mathcal{L}^{2}(\mathbf{P}, \mathbb{H})$

$$
\lim _{n \rightarrow \infty} \mathbf{E}\left\|x_{i}\left(t+s_{n}\right)-y_{i}(t)\right\|^{2}=0
$$

for each $t \in \mathbb{R}$ and $i=1,2, \cdots$.

We observe that, for each $t \in \mathbb{R}$, the sequence of $\left\{y_{i}(t)\right\}$ is a Cauchy sequence in $\mathcal{L}^{2}(\mathbf{P}, \mathbb{H})$. Indeed, if we write

$y_{i}(t)-y_{j}(t)=y_{i}(t)-x_{i}\left(t+s_{n}\right)+x_{i}\left(t+s_{n}\right)-x_{j}\left(t+s_{n}\right)+x_{j}\left(t+s_{n}\right)-y_{j}(t)$,

then we get

$$
\begin{aligned}
\mathbf{E} \| y_{i}(t) & -y_{j}(t) \|^{2} \\
\leq & 3 \mathbf{E}\left\|y_{i}(t)-x_{i}\left(t+s_{n}\right)\right\|^{2}+3 \mathbf{E}\left\|x_{i}\left(t+s_{n}\right)-x_{j}\left(t+s_{n}\right)\right\|^{2} \\
& +3 \mathbf{E}\left\|x_{j}\left(t+s_{n}\right)-y_{j}(t)\right\|^{2} .
\end{aligned}
$$

By (2.2) and (2.3), the sequence of $\left\{y_{i}(t)\right\}$ is Cauchy.

Using the completeness of the space $\mathcal{L}^{2}(\mathbf{P}, \mathbb{H})$, we denote by $y(t)$ the pointwise limit of $\left\{y_{i}(t)\right\}$. Let us prove now that

$$
\lim _{n \rightarrow \infty} \mathbf{E}\left\|x\left(t+s_{n}\right)-y(t)\right\|^{2}=0 \quad \text { and } \quad \lim _{n \rightarrow \infty} \mathbf{E}\left\|y\left(t-s_{n}\right)-x(t)\right\|^{2}=0
$$


for each $t \in \mathbb{R}$. Indeed, for each $i=1,2, \cdots$, we have

$$
\begin{aligned}
& \mathbf{E}\left\|x\left(t+s_{n}\right)-y(t)\right\|^{2} \\
& \quad \leq 3 \mathbf{E}\left\|x\left(t+s_{n}\right)-x_{i}\left(t+s_{n}\right)\right\|^{2}+3 \mathbf{E}\left\|x_{i}\left(t+s_{n}\right)-y_{i}(t)\right\|^{2}+3 \mathbf{E}\left\|y_{i}(t)-y(t)\right\|^{2} .
\end{aligned}
$$

By (2.2) and (2.3),

$$
\lim _{n \rightarrow \infty} \mathbf{E}\left\|x\left(t+s_{n}\right)-y(t)\right\|^{2}=0
$$

for each $t \in \mathbb{R}$.

We can use the same step to prove that

$$
\lim _{n \rightarrow \infty} \mathbf{E}\left\|y\left(t-s_{n}\right)-x(t)\right\|^{2}=0
$$

for each $t \in \mathbb{R}$. That is, $x(t)$ is square-mean almost automorphic. The proof is complete.

Definition 2.5. A function $f: \mathbb{R} \times \mathcal{L}^{2}(\mathbf{P}, \mathbb{H}) \rightarrow \mathcal{L}^{2}(\mathbf{P}, \mathbb{H}),(t, x) \mapsto f(t, x)$, which is jointly continuous, is said to be square-mean almost automorphic in $t \in \mathbb{R}$ for each $x \in \mathcal{L}^{2}(\mathbf{P}, \mathbb{H})$ if for every sequence of real numbers $\left\{s_{n}^{\prime}\right\}$, there exists a subsequence $\left\{s_{n}\right\}$ such that for some function $\tilde{f}$

$$
\lim _{n \rightarrow \infty} \mathbf{E}\left\|f\left(t+s_{n}, x\right)-\widetilde{f}(t, x)\right\|^{2}=0 \quad \text { and } \quad \lim _{n \rightarrow \infty} \mathbf{E}\left\|\widetilde{f}\left(t-s_{n}, x\right)-f(t, x)\right\|^{2}=0
$$

for each $t \in \mathbb{R}$ and each $x \in \mathcal{L}^{2}(\mathbf{P}, \mathbb{H})$.

Theorem 2.6. Let $f: \mathbb{R} \times \mathcal{L}^{2}(\mathbf{P}, \mathbb{H}) \rightarrow \mathcal{L}^{2}(\mathbf{P}, \mathbb{H}),(t, x) \mapsto f(t, x)$ be square-mean almost automorphic in $t \in \mathbb{R}$ for each $x \in \mathcal{L}^{2}(\mathbf{P}, \mathbb{H})$, and assume that $f$ satisfies the Lipschitz condition in the following sense:

$$
\mathbf{E}\|f(t, x)-f(t, y)\|^{2} \leq L \mathbf{E}\|x-y\|^{2}
$$

for all $x, y \in \mathcal{L}^{2}(\mathbf{P}, \mathbb{H})$ and for each $t \in \mathbb{R}$, where $L>0$ is independent of $t$. Then for any square-mean almost automorphic process $x: \mathbb{R} \rightarrow \mathcal{L}^{2}(\mathbf{P}, \mathbb{H})$, the stochastic process $F: \mathbb{R} \rightarrow \mathcal{L}^{2}(\mathbf{P}, \mathbb{H})$ given by $F(t):=f(t, x(t))$ is square-mean almost automorphic.

Proof. Let $\left\{s_{n}^{\prime}\right\}$ be a sequence of real numbers. By the almost automorphy of $f$ and $x$, we can extract a subsequence $\left\{s_{n}\right\}$ of $\left\{s_{n}^{\prime}\right\}$ such that for some function $\tilde{f}$ and for each $t \in \mathbb{R}$ and $x \in \mathcal{L}^{2}(\mathbf{P}, \mathbb{H})$,

$$
\lim _{n \rightarrow \infty} \mathbf{E}\left\|f\left(t+s_{n}, x\right)-\tilde{f}(t, x)\right\|^{2}=0,
$$

and for some function $y$ and for each $t \in \mathbb{R}$,

$$
\lim _{n \rightarrow \infty} \mathbf{E}\left\|x\left(t+s_{n}\right)-y(t)\right\|^{2}=0 .
$$

Let us consider the function $\widetilde{F}: \mathbb{R} \rightarrow \mathcal{L}^{2}(\mathbf{P}, \mathbb{H})$ defined by $\widetilde{F}(t):=\widetilde{f}(t, y(t))$, $t \in \mathbb{R}$. Note that

$$
\begin{aligned}
F\left(t+s_{n}\right)-\widetilde{F}(t)= & f\left(t+s_{n}, x\left(t+s_{n}\right)\right)-f\left(t+s_{n}, y(t)\right) \\
& +f\left(t+s_{n}, y(t)\right)-\widetilde{f}(t, y(t)),
\end{aligned}
$$


so we have

$$
\begin{aligned}
& \mathbf{E}\left\|F\left(t+s_{n}\right)-\widetilde{F}(t)\right\|^{2} \\
& \quad \leq 2 \mathbf{E}\left\|f\left(t+s_{n}, x\left(t+s_{n}\right)\right)-f\left(t+s_{n}, y(t)\right)\right\|^{2}+2 \mathbf{E}\left\|f\left(t+s_{n}, y(t)\right)-\widetilde{f}(t, y(t))\right\|^{2} \\
& \quad \leq 2 L \mathbf{E}\left\|x\left(t+s_{n}\right)-y(t)\right\|^{2}+2 \mathbf{E}\left\|f\left(t+s_{n}, y(t)\right)-\widetilde{f}(t, y(t))\right\|^{2} .
\end{aligned}
$$

We can deduce from (2.4) and (2.5) that

$$
\lim _{n \rightarrow \infty} \mathbf{E}\left\|F\left(t+s_{n}\right)-\widetilde{F}(t)\right\|^{2}=0, \text { for each } t \in \mathbb{R} .
$$

Similarly we can prove that $\lim _{n \rightarrow \infty} \mathbf{E}\left\|\widetilde{F}\left(t-s_{n}\right)-F(t)\right\|^{2}=0$ for each $t \in \mathbb{R}$, which proves the square-mean almost automorphy of $F(t)$.

\section{THE LINEAR STOCHASTIC DIFFERENTIAL EQUATIONS}

Consider the following linear stochastic differential equation

$$
\mathrm{d} x(t)=A x(t) \mathrm{d} t+f(t) \mathrm{d} t+g(t) \mathrm{d} W(t), t \in \mathbb{R},
$$

where $A$ is an infinitesimal generator which generates a $\mathcal{C}_{0}$-semigroup $\left(T(t)_{t \geq 0}\right)$ such that

$$
\|T(t)\| \leq K e^{-\omega t}, \text { for all } t \geq 0
$$

with $K>0, \omega>0$. In addition, $f: \mathbb{R} \rightarrow \mathcal{L}^{2}(\mathbf{P}, \mathbb{H}), g: \mathbb{R} \rightarrow \mathcal{L}^{2}(\mathbf{P}, \mathbb{H})$ are stochastic processes, and $W(t)$ is a two-sided standard one-dimensional Brown motion defined on the filtered probability space $\left(\Omega, \mathcal{F}, \mathbf{P}, \mathcal{F}_{t}\right)$, where $\mathcal{F}_{t}=\sigma\{W(u)-W(v) ; u, v \leq$ $t\}$.

Definition 3.1. An $\mathcal{F}_{t}$-progressively measurable process $\{x(t)\}_{t \in \mathbb{R}}$ is called a mild solution of (3.1) if it satisfies the corresponding stochastic integral equation

$$
x(t)=T(t-a) x(a)+\int_{a}^{t} T(t-s) f(s) \mathrm{d} s+\int_{a}^{t} T(t-s) g(s) \mathrm{d} W(s),
$$

for all $t \geq a$ and each $a \in \mathbb{R}$.

Theorem 3.2. Given $f, g \in A A\left(\mathbb{R} ; \mathcal{L}^{2}(\mathbf{P}, \mathbb{H})\right)$, (3.1) has a unique square-mean almost automorphic mild solution.

Proof. It is well known (see [1]) that for given $a \in \mathbb{R}$ and given initial value $x_{a}$ at 'time' $a$, the process

$$
x(t)=T(t-a) x_{a}+\int_{a}^{t} T(t-s) f(s) \mathrm{d} s+\int_{a}^{t} T(t-s) g(s) \mathrm{d} W(s), \quad t \geq a,
$$

is the unique mild solution to (3.1) with the initial value condition $x(a)=x_{a}$. So to prove the existence of a square-mean almost automorphic mild solution, we need to find an initial value $x_{a}$ such that the stochastic process given by (3.3) is square-mean almost automorphic.

Let $\left\{s_{n}^{\prime}\right\}$ be an arbitrary sequence of real numbers. Since $f$ and $g$ are squaremean almost automorphic, there exists a subsequence $\left\{s_{n}\right\}$ of $\left\{s_{n}^{\prime}\right\}$ such that for 
certain stochastic processes $\widetilde{f}$ and $\widetilde{g}$

$$
\lim _{n \rightarrow \infty} \mathbf{E}\left\|f\left(t+s_{n}\right)-\widetilde{f}(t)\right\|^{2}=0, \quad \lim _{n \rightarrow \infty} \mathbf{E}\left\|\widetilde{f}\left(t-s_{n}\right)-f(t)\right\|^{2}=0
$$

and

$$
\lim _{n \rightarrow \infty} \mathbf{E}\left\|g\left(t+s_{n}\right)-\widetilde{g}(t)\right\|^{2}=0, \quad \lim _{n \rightarrow \infty} \mathbf{E}\left\|\widetilde{g}\left(t-s_{n}\right)-g(t)\right\|^{2}=0
$$

hold for each $t \in \mathbb{R}$.

Now we consider $u(t):=\int_{-\infty}^{t} T(t-s) f(s) \mathrm{d} s$, defined as

$$
\lim _{r \rightarrow-\infty} \int_{r}^{t} T(t-s) f(s) \mathrm{d} s .
$$

From [8, Theorem 3.1], we know that $\int_{r}^{t} T(t-s) f(s) \mathrm{d} s$ exists for each $r<t$. Moreover, if we let $\widetilde{u}(t):=\int_{-\infty}^{t} T(t-s) \widetilde{f}(s) \mathrm{d} s$, we have

$$
u\left(t+s_{n}\right) \rightarrow \widetilde{u}(t) \quad \text { and } \quad \widetilde{u}\left(t-s_{n}\right) \rightarrow u(t) \quad \text { in } \mathcal{L}^{2}(\mathbf{P}, \mathbb{H}), \quad \text { as } n \rightarrow \infty
$$

for each $t \in \mathbb{R}$. This indicates that $u$ is square-mean almost automorphic. Note that $u(a)=\int_{-\infty}^{a} T(a-s) f(s) \mathrm{d} s$. If $t \geq a$, then

$$
\begin{aligned}
\int_{a}^{t} T(t-s) f(s) \mathrm{d} s & =\int_{-\infty}^{t} T(t-s) f(s) \mathrm{d} s-\int_{-\infty}^{a} T(t-s) f(s) \mathrm{d} s \\
& =u(t)-T(t-a) u(a)
\end{aligned}
$$

i.e.,

$$
u(t)=T(t-a) x(a)+\int_{a}^{t} T(t-s) f(s) \mathrm{d} s .
$$

If we choose initial value $x_{a}=u(a)$, then the process $x(t)$ given by (3.3) is squaremean almost automorphic. In fact, denote

$$
\hat{u}(t):=T(t-a) x(a)+\int_{a}^{t} T(t-s) \tilde{f}(s) \mathrm{d} s
$$

and

$$
\tilde{x}(t):=T(t-a) x(a)+\int_{a}^{t} T(t-s) \tilde{f}(s) \mathrm{d} s+\int_{a}^{t} T(t-s) \widetilde{g}(s) \mathrm{d} W(s) .
$$

Then, for each $t \in \mathbb{R}$, we have

$$
\begin{aligned}
& \lim _{n \rightarrow \infty} \mathbf{E}\left\|x\left(t+s_{n}\right)-\tilde{x}(t)\right\|^{2} \\
= & \lim _{n \rightarrow \infty} \mathbf{E} \| T\left(t+s_{n}-a\right) x(a)+\int_{a}^{t+s_{n}} T\left(t+s_{n}-s\right) f(s) \mathrm{d} s \\
+ & \int_{a}^{t+s_{n}} T\left(t+s_{n}-s\right) g(s) \mathrm{d} W(s) \\
& -T(t-a) x(a)-\int_{a}^{t} T(t-s) \widetilde{f}(s) \mathrm{d} s-\int_{a}^{t} T(t-s) \widetilde{g}(s) \mathrm{d} W(s) \|^{2} \\
= & \lim _{n \rightarrow \infty} \mathbf{E} \| u\left(t+s_{n}\right)-\hat{u}(t)+\left(\int_{-\infty}^{t+s_{n}}-\int_{-\infty}^{a}\right) T\left(t+s_{n}-s\right) g(s) \mathrm{d} W(s) \\
& -\left(\int_{-\infty}^{t}-\int_{-\infty}^{a}\right) T(t-s) \widetilde{g}(s) \mathrm{d} W(s) \|^{2} .
\end{aligned}
$$


Let $\tilde{W}(\sigma):=W\left(\sigma+s_{n}\right)-W\left(s_{n}\right)$ for each $\sigma \in \mathbb{R}$. Note that $\tilde{W}$ is also a Wiener process and has the same distribution as $W$. Hence by changing $\sigma=s-s_{n}$ and by Ito's isometry property of stochastic integrals (see [11, p. 29], for example), we have

$$
\begin{aligned}
& \mathbf{E}\left\|x\left(t+s_{n}\right)-\tilde{x}(t)\right\|^{2} \\
\leq & 3 \mathbf{E}\left\|u\left(t+s_{n}\right)-\hat{u}(t)\right\|^{2}+3 \mathbf{E}\left\|\int_{-\infty}^{t} T(t-\sigma)\left[g\left(\sigma+s_{n}\right)-\widetilde{g}(\sigma)\right] \mathrm{d} \tilde{W}(\sigma)\right\|^{2} \\
& +3 \mathbf{E}\left\|\int_{-\infty}^{a} T(t-\sigma)\left[g\left(\sigma+s_{n}\right)-\widetilde{g}(\sigma)\right] \mathrm{d} \tilde{W}(\sigma)\right\|^{2} \\
\leq & 3 \mathbf{E}\left\|u\left(t+s_{n}\right)-\hat{u}(t)\right\|^{2}+3\left[\int_{-\infty}^{t}\|T(t-\sigma)\|^{2} \mathbf{E}\left\|g\left(\sigma+s_{n}\right)-\widetilde{g}(\sigma)\right\|^{2} \mathrm{~d} \sigma\right] \\
& +3\left[\int_{-\infty}^{a}\|T(t-\sigma)\|^{2} \mathbf{E}\left\|g\left(\sigma+s_{n}\right)-\widetilde{g}(\sigma)\right\|^{2} \mathrm{~d} \sigma\right] .
\end{aligned}
$$

Noting that $g$ is square-mean almost automorphic and by the exponential dissipation property (3.2) of $T(t)$, we immediately obtain that

$$
\lim _{n \rightarrow \infty} \mathbf{E}\left\|x\left(t+s_{n}\right)-\tilde{x}(t)\right\|^{2}=0 .
$$

And we can show in a similar way that

$$
\lim _{n \rightarrow \infty} \mathbf{E}\left\|\tilde{x}\left(t-s_{n}\right)-x(t)\right\|^{2}=0
$$

for each $t \in \mathbb{R}$. So far, the existence has been proved.

We finally prove the uniqueness of the square-mean almost automorphic solution of (3.1). Assume that $x(t)$ and $y(t)$ are both square-mean almost automorphic solutions of (3.1) with different initial values $x(a)$ and $y(a)$ at 'time' $a$. That is, for $t \geq a$,

$$
\begin{aligned}
& x(t)=T(t-a) x(a)+\int_{a}^{t} T(t-s) f(s) \mathrm{d} s+\int_{a}^{t} T(t-s) g(s) \mathrm{d} W(s), \\
& y(t)=T(t-a) y(a)+\int_{a}^{t} T(t-s) f(s) \mathrm{d} s+\int_{a}^{t} T(t-s) g(s) \mathrm{d} W(s),
\end{aligned}
$$

and $x(a) \neq y(a)$. Let $z(t)=x(t)-y(t)$. Then $z(t)$ satisfies the equation

$$
\mathrm{d} z(t)=A z(t) \mathrm{d} t, \quad t \geq a,
$$

with initial condition $z(a)=x(a)-y(a)$. Hence

$$
z(t)=T(t-a) z(a)
$$

and

$$
\|z(t)\| \leq K e^{-\omega(t-a)}\|z(a)\|, \quad \text { for all } t \geq a .
$$

So $z(t) \rightarrow 0$, as $t \rightarrow+\infty$. Since $z(t) \in A A\left(\mathbb{R} ; \mathcal{L}^{2}(\mathbf{P}, \mathbb{H})\right)$, for any sequence of real numbers $\left\{s_{n}^{\prime}\right\}$, there exists a subsequence $\left\{s_{n}\right\}$ of $\left\{s_{n}^{\prime}\right\}$ such that for some progress $\tilde{z}(t)$

$$
\lim _{n \rightarrow \infty} z\left(t+s_{n}\right)=\tilde{z}(t) \quad \text { and } \quad \lim _{n \rightarrow \infty} \tilde{z}\left(t-s_{n}\right)=z(t)
$$

for each $t \in \mathbb{R}$. In particular, if $\lim _{n \rightarrow \infty} s_{n}^{\prime}=\infty$, then $\tilde{z}(t) \equiv 0$ by the first equality of (3.4). Hence $z(t) \equiv 0$ by the second equality of (3.4), so we must have $x(a)=y(a)$, a contradiction. The proof is complete. 


\section{The NON-Linear Stochastic DifFEREnTIAL EQUATIONS}

Consider the following non-linear stochastic differential equation

$$
\mathrm{d} x(t)=A x(t) \mathrm{d} t+f(t, x(t)) \mathrm{d} t+g(t, x(t)) \mathrm{d} W(t), t \in \mathbb{R},
$$

where $f: \mathbb{R} \times \mathcal{L}^{2}(\mathbf{P}, \mathbb{H}) \rightarrow \mathcal{L}^{2}(\mathbf{P}, \mathbb{H}), g: \mathbb{R} \times \mathcal{L}^{2}(\mathbf{P}, \mathbb{H}) \rightarrow \mathcal{L}^{2}(\mathbf{P}, \mathbb{H})$, and $W(t)$ is a two-sided standard one-dimensional Brown motion defined on the filtered probability space $\left(\Omega, \mathcal{F}, \mathbf{P}, \mathcal{F}_{t}\right)$, where $\mathcal{F}_{t}=\sigma\{W(u)-W(v) ; u, v \leq t\}$.

As in the previous section, we also assume that $A$ generates a $\mathcal{C}_{0}$-semigroup $\left(T(t)_{t \geq 0}\right)$ such that

$$
\|T(t)\| \leq K e^{-\omega t}, \text { for all } t \geq 0
$$

with $K>0, \omega>0$.

Definition 4.1. An $\mathcal{F}_{t}$-progressively measurable stochastic process $\{x(t)\}_{t \in \mathbb{R}}$ is called a mild solution of (4.1) if it satisfies the corresponding stochastic integral equation

$$
x(t)=T(t-r) x(r)+\int_{r}^{t} T(t-s) f(s, x(s)) \mathrm{d} s+\int_{r}^{t} T(t-s) g(s, x(s)) \mathrm{d} W(s),
$$

for all $t \geq r$ and each $r \in \mathbb{R}$.

Theorem 4.2. Assume $f$ and $g$ are square-mean almost automorphic processes in $t \in \mathbb{R}$ for each $x \in \mathcal{L}^{2}(\mathbf{P}, \mathbb{H})$. Moreover $f$ and $g$ satisfy Lipschitz conditions in $x$ uniformly for $t$; that is, for all $x, y \in \mathcal{L}^{2}(\mathbf{P}, \mathbb{H})$ and $t \in \mathbb{R}$,

$$
\begin{aligned}
& \mathbf{E}\|f(t, x)-f(t, y)\|^{2} \leq L \mathbf{E}\|x-y\|^{2}, \\
& \mathbf{E}\|g(t, x)-g(t, y)\|^{2} \leq L^{\prime} \mathbf{E}\|x-y\|^{2},
\end{aligned}
$$

for constants $L, L^{\prime}>0$. Then (4.1) has a unique square-mean almost automorphic mild solution, provided $\frac{2 K^{2} L}{\omega^{2}}+\frac{K^{2} L^{\prime}}{\omega}<1$.

Proof. By Definition 4.1, the stochastic process $x: \mathbb{R} \rightarrow \mathcal{L}^{2}(\mathbf{P}, \mathbb{H})$ is a solution to (4.1) if and only if it satisfies the stochastic integral equation

$$
x(t)=T(t-r) x(r)+\int_{r}^{t} T(t-s) f(s, x(s)) \mathrm{d} s+\int_{r}^{t} T(t-s) g(s, x(s)) \mathrm{d} W(s) .
$$

If we let $r \rightarrow-\infty$ in the above integral equation, by the exponential dissipation condition of $T$, (4.2), then we obtain that the stochastic process $x$ is a solution to (4.1) if and only if $x$ satisfies the stochastic integral equation

$$
x(t)=\int_{-\infty}^{t} T(t-s) f(s, x(s)) \mathrm{d} s+\int_{-\infty}^{t} T(t-s) g(s, x(s)) \mathrm{d} W(s) .
$$

To seek the square-mean almost automorphic mild solution, let us consider the non-linear operator $\mathcal{S}$ acting on the Banach space $A A\left(\mathbb{R} ; \mathcal{L}^{2}(\mathbf{P}, \mathbb{H})\right)$ given by

$$
(\mathcal{S} x)(t):=\int_{-\infty}^{t} T(t-s) f(s, x(s)) \mathrm{d} s+\int_{-\infty}^{t} T(t-s) g(s, x(s)) \mathrm{d} W(s) .
$$

If we can show that the operator $\mathcal{S}$ maps $A A\left(\mathbb{R} ; \mathcal{L}^{2}(\mathbf{P}, \mathbb{H})\right)$ into itself and it is a contraction mapping, then by the Banach fixed point theorem, we can conclude that there is a unique square-mean almost automorphic mild solution to the equation (4.1). 
Let us consider the non-linear operators $\mathcal{S}_{1} x$ and $\mathcal{S}_{2} x$ acting on the Banach space $A A\left(\mathbb{R} ; \mathcal{L}^{2}(\mathbf{P}, \mathbb{H})\right)$ given by

$$
\left(\mathcal{S}_{1} x\right)(t):=\int_{-\infty}^{t} T(t-s) f(s, x(s)) \mathrm{d} s
$$

and

$$
\left(\mathcal{S}_{2} x\right)(t):=\int_{-\infty}^{t} T(t-s) g(s, x(s)) \mathrm{d} W(s),
$$

respectively. By Theorem 2.6, $F_{1}(t):=f(t, x(t))$ and $F_{2}(t):=g(t, x(t))$ are squaremean almost automorphic if $x$ is; then by the proof of Theorem 3.2. we know that $\mathcal{S}_{1} x$ and $\mathcal{S}_{2} x$ are square-mean almost automorphic if $F_{1}$ and $F_{2}$ are. That is, the operator $\mathcal{S}$ maps $A A\left(\mathbb{R} ; \mathcal{L}^{2}(\mathbf{P}, \mathbb{H})\right)$ into itself.

Next we show that $\mathcal{S}$ is a contraction mapping on $A A\left(\mathbb{R} ; \mathcal{L}^{2}(\mathbf{P}, \mathbb{H})\right)$. For $x_{1}, x_{2} \in$ $A A\left(\mathbb{R} ; \mathcal{L}^{2}(\mathbf{P}, \mathbb{H})\right)$ and each $t \in \mathbb{R}$ we have

$$
\begin{aligned}
\mathbf{E}\left\|\left(\mathcal{S} x_{1}\right)(t)-\left(\mathcal{S} x_{2}\right)(t)\right\|^{2}= & \mathbf{E} \| \int_{-\infty}^{t} T(t-s)\left[f\left(s, x_{1}(s)\right)-f\left(s, x_{2}(s)\right)\right] \mathrm{d} s \\
& +\int_{-\infty}^{t} T(t-s)\left[g\left(s, x_{1}(s)\right)-g\left(s, x_{2}(s)\right)\right] \mathrm{d} W(s) \|^{2} \\
\leq & 2 K^{2} \mathbf{E}\left(\int_{-\infty}^{t} e^{-\omega(t-s)}\left\|f\left(s, x_{1}(s)\right)-f\left(s, x_{2}(s)\right)\right\| \mathrm{d} s\right)^{2} \\
& +2 \mathbf{E}\left\|\int_{-\infty}^{t} T(t-s)\left[g\left(s, x_{1}(s)\right)-g\left(s, x_{2}(s)\right)\right] \mathrm{d} W(s)\right\|^{2} .
\end{aligned}
$$

We first evaluate the first term of the right-hand side by the Cauchy-Schwarz inequality as follows:

$$
\begin{aligned}
& \mathbf{E}\left(\int_{-\infty}^{t} e^{-\omega(t-s)}\left\|f\left(s, x_{1}(s)\right)-f\left(s, x_{2}(s)\right)\right\| \mathrm{d} s\right)^{2} \\
= & \mathbf{E}\left(\int_{-\infty}^{t}\left(e^{\frac{-\omega(t-s)}{2}}\right)\left(e^{\frac{-\omega(t-s)}{2}}\right)\left\|f\left(s, x_{1}(s)\right)-f\left(s, x_{2}(s)\right)\right\| \mathrm{d} s\right)^{2} \\
\leq & \mathbf{E}\left[\left(\int_{-\infty}^{t} e^{-\omega(t-s)} \mathrm{d} s\right)\left(\int_{-\infty}^{t} e^{-\omega(t-s)}\left\|f\left(s, x_{1}(s)\right)-f\left(s, x_{2}(s)\right)\right\|^{2} \mathrm{~d} s\right)\right] \\
\leq & \left(\int_{-\infty}^{t} e^{-\omega(t-s)} \mathrm{d} s\right)\left(\int_{-\infty}^{t} e^{-\omega(t-s)} \mathbf{E}\left\|f\left(s, x_{1}(s)\right)-f\left(s, x_{2}(s)\right)\right\|^{2} \mathrm{~d} s\right) \\
\leq & L \cdot\left(\int_{-\infty}^{t} e^{-\omega(t-s)} \mathrm{d} s\right)\left(\int_{-\infty}^{t} e^{-\omega(t-s)} \mathbf{E}\left\|x_{1}(s)-x_{2}(s)\right\|^{2} \mathrm{~d} s\right) \\
\leq & L \cdot\left(\int_{-\infty}^{t} e^{-\omega(t-s)} \mathrm{d} s\right)^{2} \sup _{s \in \mathbb{R}} \mathbf{E}\left\|x_{1}(s)-x_{2}(s)\right\|^{2} \\
\leq & \frac{L}{\omega^{2}} \cdot \sup _{s \in \mathbb{R}} \mathbf{E}\left\|x_{1}(s)-x_{2}(s)\right\|^{2} .
\end{aligned}
$$


As to the second term, by Ito's isometry property of stochastic integrals, we have

$$
\begin{aligned}
\mathbf{E} & \left\|\int_{-\infty}^{t} T(t-s)\left[g\left(s, x_{1}(s)\right)-g\left(s, x_{2}(s)\right)\right] \mathrm{d} W(s)\right\|^{2} \\
& =\mathbf{E}\left[\int_{-\infty}^{t}\left\|T(t-s)\left[g\left(s, x_{1}(s)\right)-g\left(s, x_{2}(s)\right)\right]\right\|^{2} \mathrm{~d} s\right] \\
& \leq \mathbf{E}\left[\int_{-\infty}^{t}\|T(t-s)\|^{2}\left\|g\left(s, x_{1}(s)\right)-g\left(s, x_{2}(s)\right)\right\|^{2} \mathrm{~d} s\right] \\
& \leq K^{2} \int_{-\infty}^{t} e^{-2 \omega(t-s)} \mathbf{E}\left\|g\left(s, x_{1}(s)\right)-g\left(s, x_{2}(s)\right)\right\|^{2} \mathrm{~d} s \\
& \leq K^{2} L^{\prime} \cdot\left(\int_{-\infty}^{t} e^{-2 \omega(t-s)} \mathrm{d} s\right) \sup _{s \in \mathbb{R}} \mathbf{E}\left\|x_{1}(s)-x_{2}(s)\right\|^{2} \\
& \leq \frac{K^{2} L^{\prime}}{2 \omega} \cdot \sup _{s \in \mathbb{R}} \mathbf{E}\left\|x_{1}(s)-x_{2}(s)\right\|^{2} .
\end{aligned}
$$

Thus, it follows that, for each $t \in \mathbb{R}$,

$$
\mathbf{E}\left\|\left(\mathcal{S} x_{1}\right)(t)-\left(\mathcal{S} x_{2}\right)(t)\right\|^{2} \leq\left(\frac{2 K^{2} L}{\omega^{2}}+\frac{K^{2} L^{\prime}}{\omega}\right) \cdot \sup _{s \in \mathbb{R}} \mathbf{E}\left\|x_{1}(s)-x_{2}(s)\right\|^{2} ;
$$

that is,

$$
\left\|\left(\mathcal{S} x_{1}\right)(t)-\left(\mathcal{S} x_{2}\right)(t)\right\|_{2}^{2} \leq \eta \cdot \sup _{s \in \mathbb{R}}\left\|x_{1}(s)-x_{2}(s)\right\|_{2}^{2}
$$

with $\eta:=\frac{2 K^{2} L}{\omega^{2}}+\frac{K^{2} L^{\prime}}{\omega}$. Note that

$$
\sup _{s \in \mathbb{R}}\left\|x_{1}(s)-x_{2}(s)\right\|_{2}^{2} \leq\left(\sup _{s \in \mathbb{R}}\left\|x_{1}(s)-x_{2}(s)\right\|_{2}\right)^{2}
$$

and (4.3) together with (4.4) give, for each $t \in \mathbb{R}$,

$$
\left\|\mathcal{S}\left(x_{1}\right)(t)-\mathcal{S}\left(x_{2}\right)(t)\right\|_{2} \leq \sqrt{\eta}\left\|x_{1}-x_{2}\right\|_{\infty} .
$$

Hence

$$
\left\|\mathcal{S} x_{1}-\mathcal{S} x_{2}\right\|_{\infty}=\sup _{t \in \mathbb{R}}\left\|\mathcal{S}\left(x_{1}\right)(t)-\mathcal{S}\left(x_{2}\right)(t)\right\|_{2} \leq \sqrt{\eta}\left\|x_{1}-x_{2}\right\|_{\infty} .
$$

Since $\eta<1$, it follows that $\mathcal{S}$ is a contraction mapping on $A A\left(\mathbb{R} ; \mathcal{L}^{2}(\mathbf{P}, \mathbb{H})\right)$. Therefore, there exists a unique $v \in A A\left(\mathbb{R} ; \mathcal{L}^{2}(\mathbf{P}, \mathbb{H})\right)$ such that $\mathcal{S} v=v$, which is the unique solution to (4.1). The proof is now complete.

Remark 4.3. If $f$ and $g$ in (3.1) and (4.1) are almost periodic in $t$, then the unique square-mean almost automorphic solution obtained in Theorems 3.2 and 4.2 is actually almost periodic; see [2, 3, 4, 6, 9, 13].

\section{Stability of the Unique Square-mean ALMOST AUTOMORPHIC SOLUTION}

In the previous section, for the non-linear stochastic differential equation (4.1), we obtain that it has a unique square-mean almost automorphic solution. In this section, we will show that the unique square-mean almost automorphic solution is asymptotically stable in the square-mean sense and that any other solutions converge to it exponentially fast. First, let us state the definition of asymptotic stability. 
Definition 5.1. The unique square-mean almost automorphic solution $x_{a a}(t)$ of (4.1) is said to be stable in the square-mean sense if for arbitrary $\epsilon>0$, there exists $\delta>0$ such that

$$
\mathbf{E}\left\|x_{c}(t)-x_{a a}(t)\right\|^{2}<\epsilon, \quad t \geq 0,
$$

whenever $\left\|c-x_{a a}(0)\right\|^{2}<\delta$, where $x_{c}(t)$ stands for the solution of (4.1) with initial condition $x_{c}(0)=c$. The solution $x_{a a}(t)$ is said to be asymptotically stable in the square-mean sense if it is stable in the square-mean sense and

$$
\lim _{t \rightarrow \infty} \mathbf{E}\left\|x_{c}(t)-x_{a a}(t)\right\|^{2}=0 .
$$

Theorem 5.2. Assume that the assumptions of Theorem 4.2 hold, then the unique square-mean almost automorphic solution $x_{a a}(t)$ of (4.1) is asymptotically stable in the square-mean sense.

Proof. We actually will prove more general results. Assume that $x(t)$ and $y(t)$ are two solutions of (4.1) with initial values $x(0)$ and $y(0)$, respectively. Note that, by the exponential dissipation of $T(t)$, we have

$$
\begin{aligned}
\| y(t)- & x(t)\left\|^{2}=\right\| T(t)[y(0)-x(0)]+\int_{0}^{t} T(t-s)[f(s, y(s))-f(s, x(s))] \mathrm{d} s \\
& +\int_{0}^{t} T(t-s)[g(s, y(s))-g(s, x(s))] \mathrm{d} W(s) \|^{2} \\
\leq & 3 K^{2} e^{-2 \omega t}\|y(0)-x(0)\|^{2}+3\left[\int_{0}^{t} K e^{-\omega(t-s)}\|f(s, y(s))-f(s, x(s))\| \mathrm{d} s\right]^{2} \\
& +3\left[\int_{0}^{t} K e^{-\omega(t-s)}[g(s, y(s))-g(s, x(s))] \mathrm{d} W(s)\right]^{2} .
\end{aligned}
$$

Since $f$ satisfies the Lipschitz condition, using the Cauchy-Schwarz inequality, we have

$$
\begin{aligned}
& 3\left[\int_{0}^{t} K e^{-\omega(t-s)}\|f(s, y(s))-f(s, x(s))\| \mathrm{d} s\right]^{2} \\
\leq & 3 K^{2} L^{2}\left(\int_{0}^{t} e^{-\omega(t-s)}\|y(s)-x(s)\| \mathrm{d} s\right)^{2} \\
= & 3 K^{2} L^{2}\left(\int_{0}^{t}\left(e^{\frac{-\omega(t-s)}{2}}\right)\left(e^{\frac{-\omega(t-s)}{2}}\right)\|y(s)-x(s)\| \mathrm{d} s\right)^{2} \\
\leq & 3 K^{2} L^{2}\left(\int_{0}^{t} e^{-\omega(t-s)} d s\right)\left(\int_{0}^{t} e^{-\omega(t-s)}\|y(s)-x(s)\|^{2} d s\right) \\
\leq & \frac{3 K^{2} L^{2}}{\omega} \int_{0}^{t} e^{-\omega(t-s)}\|y(s)-x(s)\|^{2} \mathrm{~d} s,
\end{aligned}
$$

for all $t \geq 0$. By Ito's isometry property of stochastic integrals, it follows that

$$
\begin{aligned}
& 3\left[\int_{0}^{t} K e^{-\omega(t-s)}[g(s, y(s))-g(s, x(s))] \mathrm{d} W(s)\right]^{2} \\
\leq & 3 K^{2} L^{\prime 2} \int_{0}^{t} e^{-2 \omega(t-s)}\|y(s)-x(s)\|^{2} \mathrm{~d} s
\end{aligned}
$$


for all $t \geq 0$.

Let $Y(t):=\mathbf{E}\|y(t)-x(t)\|^{2}$ and $k:=3 K^{2} \hat{L}^{2}\left(1+\frac{1}{\omega}\right)$ with $\hat{L}:=\max \left\{L, L^{\prime}\right\}$. Note that $e^{-2 \omega t} \leq e^{-\omega t}$ for $t \geq 0$ and by (5.1), (5.2), (5.3), we have

$$
Y(t) \leq 3 K^{2} e^{-\omega t} Y(0)+k \int_{0}^{t} e^{-\omega(t-s)} Y(s) \mathrm{d} s .
$$

The $Y(t)$ in inequality (5.4) can be controlled by $\widetilde{Y}(t)$, which satisfies

$$
\begin{aligned}
\dot{\tilde{Y}}(t) & =-\omega \tilde{Y}(t)+k \widetilde{Y}(t), \\
\tilde{Y}(0) & =3 K^{2} Y(0) .
\end{aligned}
$$

Hence $\tilde{Y}(t) \rightarrow 0$ exponentially fast if $-\omega+k<0$, that is, if

$$
\omega>3 K^{2} \hat{L}^{2}\left(1+\frac{1}{\omega}\right) \text {. }
$$

Note that (5.5) holds if and only if

$$
\omega^{2}>3 K^{2} \hat{L}^{2}(\omega+1)
$$

which always holds. Therefore, $Y(t)$ converges to 0 exponentially fast.

In particular, if we set $x(t)=x_{a a}(t)$ in the above arguments, we obtain that the unique square-mean almost automorphic solution $x_{a a}(t)$ of (4.1) is asymptotically stable in the square-mean sense. The proof is complete.

Remark 5.3. By the proof of the stability theorem, we actually obtain that any solution of (4.1) (and hence (3.1)) is asymptotically stable in the square-mean sense.

\section{ACKNOWLEDGMENTS}

The authors are indebted to Professor Yong Li for his encouragement and helpful discussions. The authors would like to thank the anonymous referee for a very careful reading of the manuscript and valuable suggestions which greatly improved the paper.

\section{REFERENCES}

[1] L. Arnold, Stochastic differential equations: theory and applications. Translated from the German. Wiley-Interscience [John Wiley \& Sons], New York-London-Sydney, 1974. MR0443083 (56:1456)

[2] L. Arnold and C. Tudor, Stationary and almost periodic solutions of almost periodic affine stochastic differential equations. Stochastics Stochastics Rep. 64 (1998), 177-193. MR.1709282 (2000d:60098)

[3] P. H. Bezandry and T. Diagana, Existence of almost periodic solutions to some stochastic differential equations. Appl. Anal. 86 (2007), 819-827. MR2355540 (2008i:60089)

[4] P. H. Bezandry and T. Diagana, Square-mean almost periodic solutions nonautonomous stochastic differential equations. Electron. J. Differential Equations (2007), No. 117. MR 2349945 (2009e:34171)

[5] S. Bochner, Curvature and Betti numbers in real and complex vector bundles. Univ. e Politec. Torino. Rend. Sem. Mat. 15 (1955-56), 225-253. MR0084160 (18:819c)

[6] G. Da Prato and C. Tudor, Periodic and almost periodic solutions for semilinear stochastic equations. Stochastic Anal. Appl. 13 (1995), 13-33. MR.1313204(96e:60112)

[7] G. M. N'Guérékata, Almost automorphic and almost periodic functions in abstract spaces. Kluwer Academic/Plenum Publishers, New York, 2001. MR.1880351 (2003d:43001) 
[8] G. M. N'Guérékata, Existence and uniqueness of almost automorphic mild solutions to some semilinear abstract differential equations. Semigroup Forum 69 (2004), 80-86. MR2063980 (2005b:34119)

[9] A. Halanay, Periodic and almost periodic solutions to affine stochastic systems. Proceedings of the Eleventh International Conference on Nonlinear Oscillations (Budapest, 1987), 94-101, János Bolyai Math. Soc., Budapest, 1987. MR933583 (89f:34085)

[10] R. A. Johnson, A linear, almost periodic equation with an almost automorphic solution. Proc. Amer. Math. Soc. 82 (1981), 199-205. MR609651 (82i:34044a)

[11] B. Øksendal, Stochastic differential equations. An introduction with applications. Sixth edition. Universitext. Springer-Verlag, Berlin, 2003. MR2001996 (2004e:60102)

[12] W. Shen and Y. Yi, Almost automorphic and almost periodic dynamics in skew-product semiflows. Mem. Amer. Math. Soc. 136 (1998), no. 647. MR1445493 (99d:34088)

[13] C. Tudor, Almost periodic solutions of affine stochastic evolution equations. Stochastics Stochastics Rep. 38 (1992), 251-266. MR1274905(95e:60058)

[14] C. A. Tudor and M. Tudor, Pseudo almost periodic solutions of some stochastic differential equations. Math. Rep. (Bucur.) 1(51) (1999), 305-314. MR1825773 (2002a:60100)

[15] W. A. Veech, Almost automorphic functions on groups. Amer. J. Math. 87 (1965), 719-751. MR0187014 (32:4469)

[16] W. A. Veech, Topological dynamics. Bull. Amer. Math. Soc. 83 (1977), 775-830. MR0467705 $(57: 7558)$

College of Mathematics, Jilin University, Changchun 130012, People's Republic of China - And - School of Mathematics, Changchun Normal College, Changchun 130032, People's Republic of China

E-mail address: mmfucaathy@yahoo.com.cn

College of Mathematics, Jilin University, Changchun 130012, People's Republic of CHINA

E-mail address: zxliu@jlu.edu.cn 